\title{
Interaction aware trajectory planning for merge scenarios in congested traffic situations
}

\author{
Niclas Evestedt, Erik Ward, John Folkesson and Daniel Axehill \\ Book Chapter \\ Tweet
}

N.B.: When citing this work, cite the original article.

Part of: 2016 IEEE 19th International Conference on Intelligent Transportation Systems

(ITSC), 2016, pp. 465-472. ISBN: 9781509018895 (online), 9781509018888 (USB), 9781509018901 (PoD)

Series: Intelligent Transportation Systems, eISSN 2153-0017, No. 2016

DOI: http://dx.doi.org/10.1109/ITSC.2016.7795596

Copyright: Institute of Electrical and Electronics Engineers (IEEE)

Available at: Linköping University Electronic Press

http://urn.kb.se/resolve?urn=urn:nbn:se:liu:diva-134034 


\title{
Interaction aware trajectory planning for merge scenarios in congested traffic situations
}

\author{
Niclas Evestedt ${ }^{1}$, Erik Ward ${ }^{2}$, John Folkesson ${ }^{2}$, Daniel Axehill ${ }^{1}$
}

\begin{abstract}
In many traffic situations there are times where interaction with other drivers is necessary and unavoidable in order to safely progress towards an intended destination. This is especially true for merge manoeuvres into dense traffic, where drivers sometimes must be somewhat aggressive and show the intention of merging in order to interact with the other driver and make the driver open the gap needed to execute the manoeuvre safely. Many motion planning frameworks for autonomous vehicles adopt a reactive approach where simple models of other traffic participants are used and therefore need to adhere to large margins in order to behave safely. However, the large margins needed can sometimes get the system stuck in congested traffic where time gaps between vehicles are too small. In other situations, such as a highway merge, it can be significantly more dangerous to stop on the entrance ramp if the gaps are found to be too small than to make a slightly more aggressive manoeuvre and let the driver behind open the gap needed. To remedy this problem, this work uses the Intelligent Driver Model (IDM) to explicitly model the interaction of other drivers and evaluates the risk by their required deceleration in a similar manner as the Minimum Overall Breaking Induced by Lane change (MOBIL) model that has been used in large scale traffic simulations before. This allows the algorithm to evaluate the effect on other drivers depending on our own trajectory plans by simulating the nearby traffic situation. Finding a globally optimal solution is often intractable in these situations so instead a large set of candidate trajectories are generated that are evaluated against the traffic scene by forward simulations of other traffic participants. By discretization and using an efficient trajectory generator together with efficient modelling of the traffic scene real-time demands can be met.
\end{abstract}

\section{INTRODUCTION}

Merging into fast highway traffic or congested roads faces drivers with some of the riskiest and most critical maneuvers that have to be performed during normal driving conditions. The driver constantly needs to assess the situation by predicting the reactions of other traffic participants while performing a fine balance between the risk of the maneuver and its own progress. Being too aggressive can result in dangerous situations or even accidents while being too passive can leave a driver waiting for a long time before a chance to merge appears. In these situations it is important to anticipate or predict the reactions of other traffic participants as they can help to create the gap needed to finish the maneuver. In previous work, such as [1] and

The research leading to these results has been carried out within the iQMatic project funded by FFI/VINNOVA. The first two authors contributed equally to this work.

${ }^{1}$ Division of Automatic Control, Linköping University, Sweden, (e-mail: niclas.evestedtaliu.se, daniel.axehilleliu.se)

${ }^{2}$ Computer Vision and Active Perception Lab, KTH, Stockholm, Sweden (e-mail: ewardekth.se, johnf@kth.se)

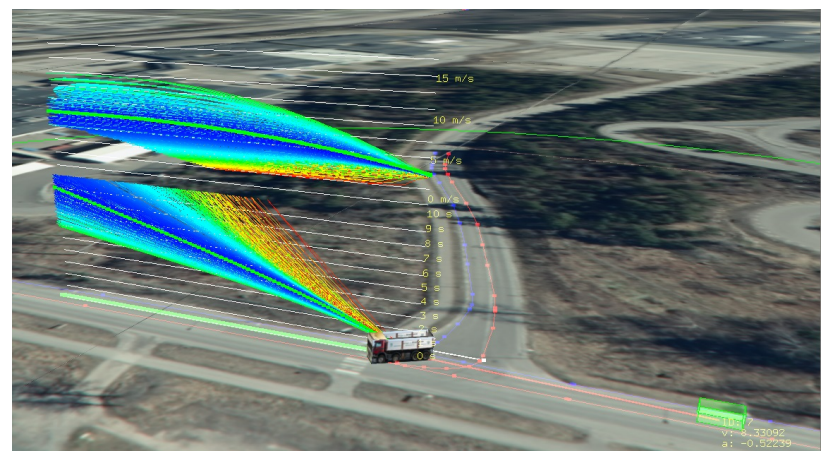

Fig. 1: Merging scenario in a T-junction. Other vehicles are represented with green boxes and the available trajectories at this planning cycle are projected along the road reference and visualized with different colors representing the cost of the trajectory. Blue means low cost and red high cost and the green trajectory is the chosen best trajectory in this planning cycle.

[2], constant velocity models have been assumed for other traffic participants and it has been shown to work for simple scenarios in simulations but in tight heads to tail traffic it can be impossible to merge without affecting the traffic flow. When considering heavy vehicles such as trucks this problem is even more pronounced due to the slow acceleration and size of the vehicle. When using constant velocity models extremely large time gaps would be required to merge and instead more realistic models of other traffic participants are needed. In this work we present a real time capable planning framework for merging into complicated heads to tail traffic by using the Intelligent Driver Model (IDM) [3], extended with information about the underlying road structure, for simulation based predictions of the traffic scene. A broad set of minimum jerk candidate velocity profiles are generated for the ego vehicle and the traffic scene is predicted for every trajectory and then evaluated and scored according to a cost function incorporating both comfort and risk. Instead of explicitly looking at time gap constraints the risk of a maneuver is captured in the resulting simulated accelerations of other traffic participants in a similar way as presented in [4]. The framework is then thoroughly tested in simulations with randomly generated traffic in two different merging scenarios.

\section{A. Related work}

Planning and decision making for autonomous vehicles in urban traffic has received a lot of attention during the last 
decade. Early work included rule-based finite state machines that considered conservative time gaps and critical sections but they quickly reached their limits when more complex traffic situations were considered [5], [6], [1]. Werling et al. [7] presented a trajectory based planning approach capable of handling more complex situations by calculating a set of candidate trajectories to a discretized terminal manifold and then selecting the most suitable trajectory within the set. The method used a short planning horizon of $3 \mathrm{~s}$ and handed the long term safety requirement of the vehicle to another decision layer on top making it unsuitable for safe merging where longer horizons are needed.

Ziegler et al. [8] presented a local optimization based trajectory generation framework that was used to successfully drive the Bertha-Benz-Memorial-Route fully autonomously. The algorithm solves the problem directly in continuous space with a cost function that incorporates several comfort and distance cost terms and dynamic obstacles are handled by creating polygon shaped constraints at different time instances along the trajectory. Even though a continuous solution manifold is preferred the optimization solver can not handle the discrete decisions that has to be made in many situations and the solution can get trapped in a local minima or even in an invalid state.

All of the above algorithms have assumed a constant velocity model for other traffic participants which has proved to work in some simpler traffic scenarios but in more complicated situations with heavy traffic and small time gaps this assumption can be too conservative making it impossible for the algorithms to decide to perform a merge. When merging with heavy trucks very large time gaps would be needed to be able to plan a merge without any effect on other traffic participants and truck drivers often have to perform aggressive merges where other vehicles have to slow down significantly to leave room for the bigger vehicle. To be able to model the social behavior in such situations within a planning framework more advanced models are needed. Wie el al. [9] used a distance keeping model for other participants and then running a prediction engine for a set of constant acceleration candidate velocity profiles to determine the most suitable one. However, since constant acceleration profiles are used big jerk values appear when switching between two acceleration sections making it uncomfortable for passengers. Another interesting approach is presented in [10] where every vehicle is given a set of possible high level actions or policies to perform and then a search for the best policy is performed by simulating the most likely traffic scenario against the available policies for the ego vehicle. Every policy is then scored against a cost function and the best policy is returned. Reducing the problem to a search over policies is an interesting concept but when merging into dense traffic, complicated policies are needed to find the best trajectory for a merge and will increase the computation time even further.

For realistic large scale traffic simulations the Intelligent Driver Model was introduced in [3] and has been shown to model collective driving behavior of real drivers quite well. Another driver model is the Foresighted Driver Model presented in [2] where drivers are modeled with a risk vs utility behavior and risk is predicted from future possible collisions. In this work we will use the IDM for modeling and prediction of future trajectories for other vehicles due to its simplicity and its ability to capture real driver behavior. However, we extend the model to include information of the underlying road network making it possible to handle interaction at intersections. We then generate a vast set of minimal jerk velocity profiles in a similar way as in [7] that are checked against the physical constraints of the ego vehicle and then fed to a prediction engine where the traffic development due to our action is predicted. Dangerous velocity profiles are then removed by checking constraints on the induced acceleration needed by other traffic participants to avoid a collision. The remaining velocity profiles are then scored according to comfort and progress levels of the ego vehicle and the risk of a maneuver is then fully captured in the induced traffic disturbance by our action measured by the needed breaking acceleration of other participants. The maximum allowed deceleration of other traffic participants introduces a parameter for an intuitive way of changing the "politeness/aggressiveness" of the algorithm. Setting it low will not allow for trajectories that have a significant impact on the traffic flow while setting it high makes it possible to force our vehicle out on a congested road while still having a measure for the predicted risk which would have been impossible if simpler models are used. Using the predicted deceleration during interactions to evaluate the risk and safety of a manoeuvre is inspired by the Minimum Overall Breaking Induced by Lane Change (MOBIL) model that has been used in large scale traffic simulations to simulate lane change behaviour [4].

The outline of the remainder of the paper is as follows: In Section II an overview of the algorithm and details of the models used and trajectory generation are given. Section III presents the simulations performed for evaluating the proposed method and finally, Section IV and Section V present the results and conclusions, respectively.

\section{INTERACTION AWARE MOTION PLANNING}

The aim of the algorithm is to find a suitable trajectory in every planning cycle that minimizes the effect on the traffic flow to ensure safety of the maneuver while using control inputs that are comfortable for passengers in the ego vehicle. Solving this problem to a global optimum is often intractable so here a three step simulation based approach is used to find the best solution within a discretized set of candidate trajectories. An overview of the steps of the algorithm can be seen in Algorithm 1. In the first step a large number of candidate trajectories are generated using a minimum jerk trajectory generator that is further described in section II-A. Once the set is created each candidate trajectory is fed into the prediction engine, described further in Section II-B below, where the behavior of all other agents are modeled using a variant of the Intelligent Driver Model (IDM) which allows for the prediction of the development 


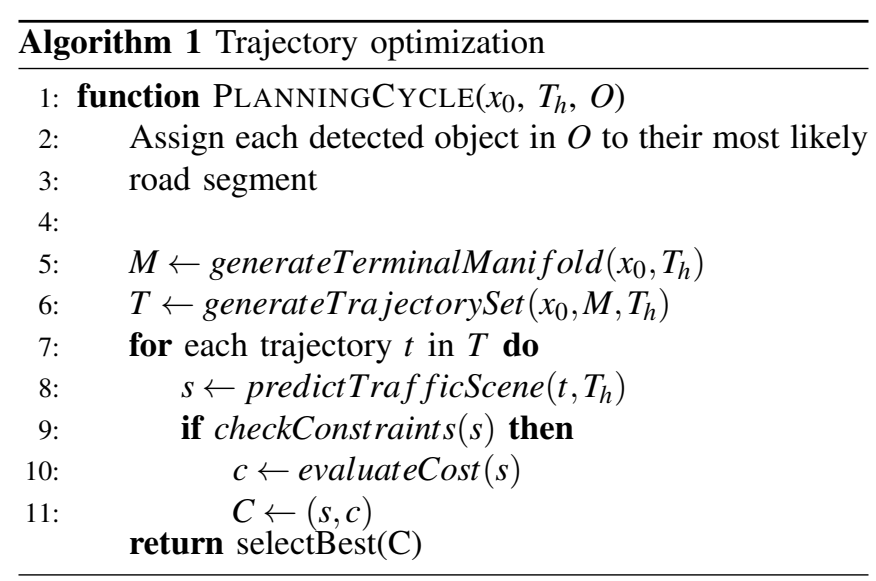

of the traffic scene depending on our own chosen trajectory. Finally, all trajectories are scored according to the cost function, discussed in Section II-C, which takes into account the risk induced by every candidate trajectory, the safety of the maneuver and the comfort in the ego vehicle. This process of candidate trajectory generation and evaluation through simulation to find the best trajectory is repeated in every planning cycle in order to adapt for new sensor data.

Our own path, and those of other traffic participants, are assumed to follow the center of a driving corridor, which in this case is defined as the center line of the road given from a road network database. By calculating intersection points of different lanes within the road network the problem can essentially be reduced to one spatial dimension by converting it into a 1-dimension arc-length representation. This allows us to calculate distances between agents efficiently using look-up tables where the distance from every agent to the critical section where two roads merge is stored. The look-up table needs only be computed once every planning cycle and can be re-used when performing simulations for all candidate trajectories.

\section{A. Trajectory generation}

To generate the candidate velocity profiles, minimal jerk solutions are calculated in a similar way as in [7]. The longitudinal movement of the ego vehicle is modeled as a double integrator system with states, $x=[s, v, a]^{T}$ where $s$ is the traveled arc-length, $v$ is the velocity and $a$ is the acceleration of the ego vehicle. The system describing the evolution of the states are given by

$$
\dot{x}=\left[\begin{array}{lll}
0 & 1 & 0 \\
0 & 0 & 1 \\
0 & 0 & 0
\end{array}\right] x+\left[\begin{array}{l}
0 \\
0 \\
1
\end{array}\right] u
$$

where the input $u$ is the longitudinal jerk. Defining the cost functional

$$
\mathscr{J}=\frac{1}{2} \int_{0}^{t_{f}} u^{2} d t
$$

and solving for the optimal minimum jerk trajectory from an initial state $x_{0}=\left[s_{0}, v_{0}, a_{0}\right]^{T}$ to a final state $x_{f}=\left[s_{f}, a_{f}\right]$ with arbitrary end velocity we get an analytical polynomial solution on the form

$$
x(t)=\left[\begin{array}{cccccc}
1 & t & t^{2} & t^{3} & t^{4} & t^{5} \\
0 & 1 & 2 t & 3 t^{2} & 4 t^{3} & 5 t^{4} \\
0 & 0 & 2 & 6 t & 12 t^{2} & 20 t^{3}
\end{array}\right]\left[\begin{array}{c}
c 1 \\
c 2 \\
c 3 \\
c 4 \\
c 5 \\
c 6
\end{array}\right]
$$

where the coefficients $c_{1-6}$ can be obtained from the initial condition $x_{0}$, the final condition $x_{f}$ and the final time $t_{f}$. By creating a discretized terminal manifold with zero final acceleration, a big set of candidate velocity profiles can be efficiently generated reaching different longitudinal positions at different times. By selecting a suitable planning horizon, $T_{h}$, and a discretization for the final time and for the final position a spatio-temporal grid of possible trajectories is constructed that can be searched for the best trajectory. To give a fair cost evaluation between all trajectories they need to have the same temporal length so all generated trajectories are extended with constant velocity from $t_{f}$ to the final horizon time $T_{h}$. The trajectories are then checked against physical constraints on the maximum acceleration, $a_{\max }$, the maximum deceleration, $a_{\min }$ and the maximum lateral acceleration $a_{\text {lat, } \max }$. The lateral acceleration is calculated using the curvature of the reference path received from the driving corridor. An example trajectory set can be seen in Fig. 2 where a temporal discretization of $1 \mathrm{~s}$ between 0 to $10 \mathrm{~s}$ and a spatial discretization of 5 meters between 0 and 50 meters have been used. Once the physically feasible trajectories have been determined they are checked against the traffic scene in the prediction step.

\section{B. Prediction engine}

Given a candidate trajectory for our own vehicle, the goal of the prediction engine is to predict the future trajectory of nearby vehicles by running a simulation of the traffic scene. The Intelligent Driver Model is used to model driver
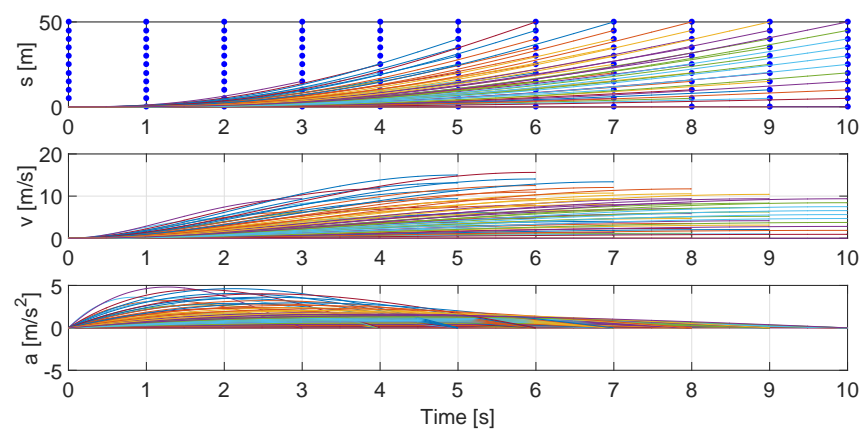

Fig. 2: Generated trajectories given the discretized end points shown as blue dots. Top graph shows the resulting arc length, middle shows the resulting velocity and the bottom shows the acceleration profile. Trajectories with $a>5.0 \mathrm{~m} / \mathrm{s}^{2}$ have been discarded. 
behaviour where each driver is controlled by a combination of two forces: one that tries to maintain a desired free cruising speed and one that tries to maintain a safe distance and time gap to the vehicle in front. Although computationally simple and not directly modelling reaction times the IDM has been shown to model different traffic situations well [3], both in large scale traffic simulations but also on a microscopic driver level where it has been used in safety assessment for advanced driver assistance systems [11]. Given a trajectory for the ego vehicle the response of all participants within the sensor range are predicted using the IDM and their predicted trajectories are later used to assess the risk of a certain maneuver. The longitudinal velocity of a vehicle is modeled according to a differential equation in the form

$$
\begin{aligned}
\dot{v} & =a\left(1-\left(\frac{v}{v_{0}}\right)^{4}-\left(\frac{d^{*}(v, \Delta v)}{d}\right)^{2}\right) \\
d^{*}(v, \Delta v) & =d_{0}+T v+\frac{v \Delta v}{2 \sqrt{a b}}
\end{aligned}
$$

where the distance $d=\left|s_{f}-s\right|$ and the velocity difference $\Delta v=v_{f}-v$ is calculated between the ego vehicle and the leading vehicle in front. From now on the vehicle in front will be referred to as the leader for another vehicle when it is used for calculations in the IDM model. The parameters $d_{0}, v_{0}, T$, $a$ and $b$ represent the minimal distance gap, the free cruising speed, the minimal time gap, the maximal acceleration and the comfortable breaking deceleration, respectively. If the distance, $d$, is large between the vehicles the first term will dominate and an adjustment of the velocity to the free cruising speed, $v_{0}$, with acceleration $a$ is initiated. On the other hand if $d$ is small or the relative velocity difference $\Delta v$ is large a deceleration of $b \mathrm{~m} / \mathrm{s}^{2}$ is initiated until the desired time gaps and distances are reached.

To extend the IDM to handle intersection and merging scenarios the underlying road network is used to determine which traffic participant that should be used as a leader in the calculations of the IDM. Lateral movements are omitted and the problem is converted to a 1-D arc-length representation by calculating a critical section where two roads intersect. In this way it is possible to keep track of when two vehicles are in a follower/leader scenario or not by checking if the vehicles have passed the critical section. Figure 3 shows the critical sections for the two scenarios used for evaluation experiments. During the prediction step the velocity profiles and position of the ego vehicle are checked against the critical section and when the crossing happens the oncoming traffic participants will acknowledge the ego vehicle as the leader and calculate its response according to the IDM. In this way the predicted evolution of the traffic scene can be predicted for every candidate trajectory and the resulting trajectories of other traffic participants can be used to evaluate the safety of a given maneuver. This opens up for possibilities to change the "politeness/aggressiveness"behavior of the algorithm in a way that is not possible with standard constant velocity models.

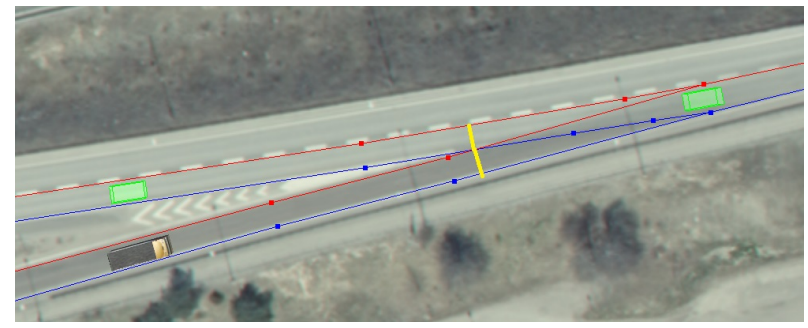

(a)

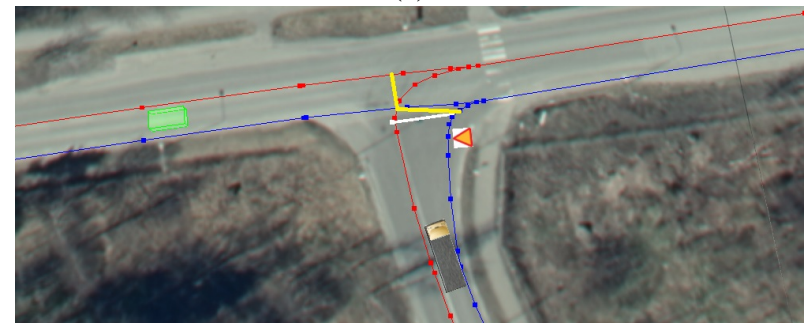

(b)

Fig. 3: Two scenarios used for evaluation. The road network is visualized by the red and blue lane markings and the begining of the critical section is draw in yellow: (a) High velocity highway merge scenario and (b) Give way Tjunction merge scenario

\section{Cost function}

Every trajectory is first checked against a set of constraints before they are evaluated according to a cost function consisting of several parts relating to safety, progress and comfort. First, the physical constraints for the maximum acceleration, $a_{\max }$, the maximum deceleration, $a_{\min }$ and the maximum lateral acceleration $a_{\text {lat, } \max }$ relating to the ego vehicle are checked.

$$
\begin{aligned}
a_{\min }<a & <a_{\max } \\
\left|a_{\text {lat }}\right| & <a_{\text {lat,max }}
\end{aligned}
$$

Next a set of safety constraints on the resulting predictions are checked. The predicted maximum deceleration, $a_{\text {follower }}$, induced on the following vehicle, the time gap, $t_{\text {lead }}$ and the minimum distance, $d_{l e a d}$, to the leading vehicle and the time gap, $t_{\text {follower }}$, to the following vehicle are constrained to

$$
\begin{aligned}
a_{\text {follower }} & >a_{\text {follower,min }} \\
t_{\text {lead }} & >t_{\text {lead,min }} \\
d_{\text {lead }} & >d_{\text {lead,min }} \\
t_{\text {follower }} & >t_{\text {follower,min }}
\end{aligned}
$$

Once the feasible trajectories have been determined they are scored according to a cost function on the form

$$
J[x(t)]=j_{\text {progress }}+j_{a_{\text {lat }}}+j_{\text {acc }}+j_{\text {gap }}+j_{\text {interaction }}
$$

where each term represents a cost for progress, comfort or safety. Each term is normalized to values between $[0,1]$ and have an individual weight, $w$, making it possible to tune the behavior for different situations in an intuitive way. The first 
term is defined as

$$
j_{\text {progress }}=w_{\text {progress }}\left(1-\frac{s\left(T_{h}\right)}{v_{0} T_{h}}\right)^{2}
$$

where $s\left(T_{h}\right)$ is the traveled arc-length at the end of the horizon for the trajectory being evaluated and $v_{0} T_{h}$ is the distance traveled when following the desired cruising speed $v_{0}$ during the full horizon time $T_{h}$. In this way the selection prefer trajectories that make maximum longitudinal progress while keeping to the speed limit. The next two terms act against the above progress term and relate to the comfort of the trajectory. The maximum lateral and longitudinal accelerations are normalized against the constraints giving the cost terms

$$
\begin{aligned}
& j_{a_{\text {lat }}}=w_{a_{\text {lat }}}\left(\frac{\left\|a_{\text {lat }}\right\|_{\infty}}{a_{\text {lat }, \text { max }}}\right)^{2} \\
& j_{\text {acc }}=w_{\text {acc }}\left(\frac{\|a\|_{\infty}}{a_{\max }}\right)^{2}
\end{aligned}
$$

To keep a safe time gap to the vehicle in front when driving on a road the following term is introduced

$$
I_{\text {gap }}=\int_{0}^{T_{h}} t_{\text {lead }}(t) d t
$$

and then a cost term on the following form can be introduced

$$
j_{\text {gap }}= \begin{cases}w_{\text {gap }}\left(\frac{t_{\text {ref }} T_{h}-I_{\text {gap }}}{T_{h}\left(t_{\text {ref }}-t_{\text {lead }, \text { min }}\right)}\right)^{2}, & \text { if } I_{\text {gap }}>t_{\text {ref }} T_{h} \\ 0, & \text { otherwise }\end{cases}
$$

This term will make the vehicle open a larger time gap if the current time gap $t_{\text {lead }}$ is smaller than the desired time gap $t_{\text {ref }}$ and have no effect if the current time gap is larger. The final term relates to the induced traffic disturbance of our trajectory, measured by the predicted acceleration response of the vehicle the ego car pulls out in front. The term is defined as

$$
j_{\text {interaction }}=w_{\text {interaction }}\left(\frac{\left\|a_{\text {follower }}\right\|_{\infty}}{a_{\text {follower }, \text { min }}}\right)^{2}
$$

and favours trajectories with the least disturbance. By selecting the constraints and weights in a suitable way a good trade-off between comfort, safety and progress can be achieved and because the reactions of other traffic participants are accounted for in the cost function the algorithm can merge safely in situations where a constant velocity model can not. In Fig. 2 a set of evaluated trajectories are shown and the color represents the total cost for each trajectory.

\section{Simulations}

To evaluate the interaction aware motion planning framework two different types of simulated merge scenarios have been created: one scenario in a busy T-junction, (TJ), and one scenario at an entrance ramp onto a busy highway, (HM). The simulated scenarios are based on real public roads in Sweden and a top-down view can be seen in Fig. 3. The highway scenario features a short on-ramp with no additional lane dedicated for merging which forces drivers to take an early
TABLE I: Statistics of front to rear distances, $d$, for simulated traffic flow with different inital vehicle distance ranges and sampled model parameters.

\begin{tabular}{lcc} 
HM & $d_{i v} \in[30,60]$ & $d_{i v} \in[30,180]$ \\
\hline mean $(d)[m]$ & 59.96 & 127.13 \\
$\operatorname{stddev}(d)[m]$ & 12.10 & 47.45 \\
TJ & $d_{i v} \in[30,50]$ & $d_{i v} \in[30,90]$ \\
\hline mean $(d)[m]$ & 34.01 & 55.33 \\
$\operatorname{stddev}(d)[m]$ & 4.76 & 16.02
\end{tabular}

decision. The speed limits for the T-junction and highway scenario are $50 \mathrm{~km} / \mathrm{h}$ and $90 \mathrm{~km} / \mathrm{h}$ respectively. Different traffic situations are created by simulating all traffic participants using the IDM with individually randomly sampled model parameters. Vehicles are added to the simulation at a specific entrance point and removed from it when they reach an exit point. By controlling the frequency of when vehicles are added we can simulate different traffic conditions, ranging from light traffic where large gaps are available for merging to dense traffic where a suitable gap can be significantly harder to find. Even though the IDM is used both to simulate the traffic flow and used for predictions within the prediction framework the random parameter selection makes sure that the parameters used by other vehicles are not known by the motion planning framework in advance making the simulations more realistic. To test different levels of congestion the vehicles are added at the entrance point when a sufficient distance to the previous vehicle, $d_{i v}$, has been achieved. After every new added vehicle the spawning distance for the next vehicle is uniformly selected in the range $\left[d_{i v, l o w e r}, d_{i v, u p p e r}\right]$. Depending on the spawning distance and the parameters drawn for different traffic participants significantly different and unique traffic conditions can be achieved. Table I shows the mean and standard deviation of intra vehicle distances, $d$, for the traffic flow simulated for some of the different $d_{i v}$, ranges used in our simulations.

In our evaluation a comparison between the interaction aware trajectory planner with a baseline minimum-time gap model which uses the same generated candidate trajectories as the interaction aware motion planner but uses a prediction model where other vehicles are assumed to continue with constant velocity and a cost function that does not consider our predicted effect on decelerations are also performed. Since vehicles have no predicted decelerations the viability of a merge maneuver is instead evaluated solely using time-gap constraints. The two algorithms are evaluated using different settings for the parameters that controls the aggressiveness of the merge maneuver, for the proposed method $a_{\text {follower,min }}$ and for the baseline method $t_{\text {follower,min }}$.

\section{A. Simulation setup}

The simulations has been performed using a single core on an Intel i7 Core(TM) i7-5930K CPU @ 3.50GHz. The planning framework is running at $10 \mathrm{~Hz}$ with a prediction horizon, $T_{h}$ of $10 \mathrm{~s}$ and the trajectory generation and predictions are using a time step, $T_{s}$ of $0.1 \mathrm{~s}$. The trajectory generator uses a time discretisation between 0 and $T_{h}$ with 


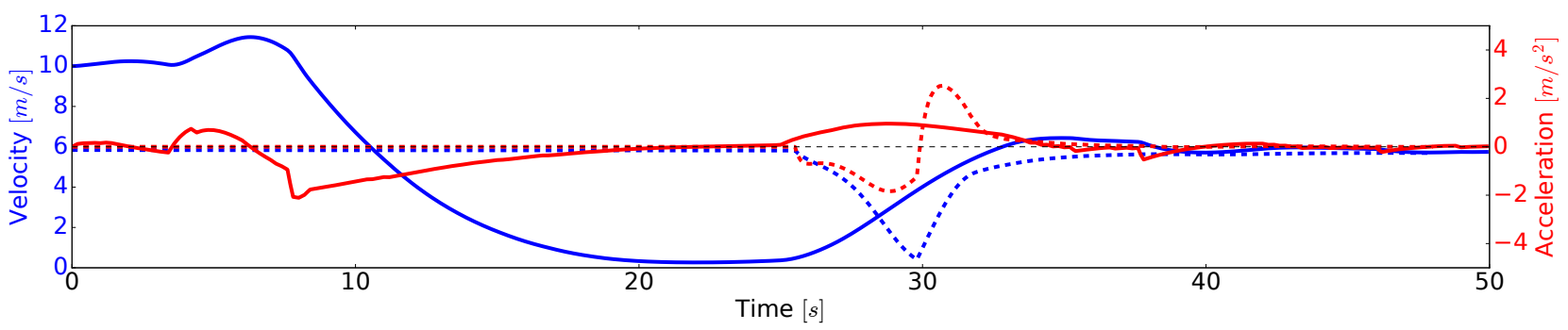

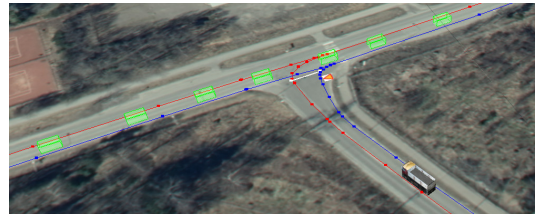

$t=7.7 s$

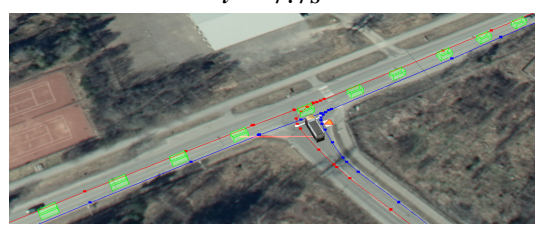

$t=25.5 \mathrm{~s}$

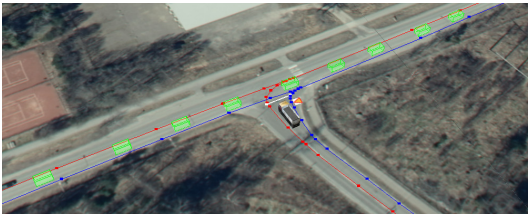

$t=16.7 \mathrm{~s}$

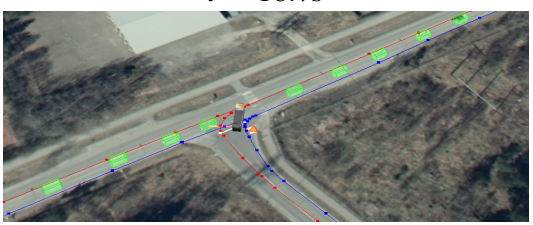

$t=29.6 s$

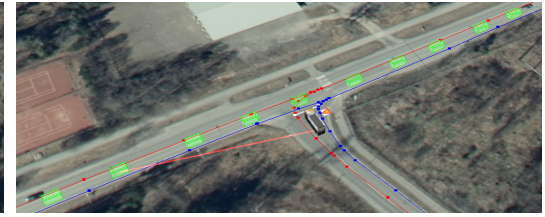

$t=19.1 s$

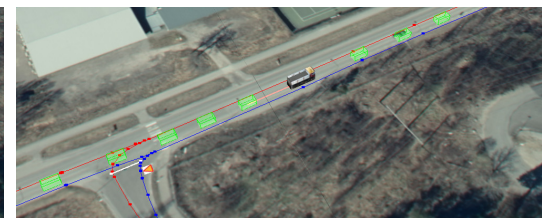

$t=39.1 s$

Fig. 4: T-junction merge into slow moving dense traffic with $a_{\text {follower,min }}=-3 \mathrm{~m} / \mathrm{s}^{2}$. Solid lines represent velocity and acceleration profiles for the ego vehicle and dashed lines represent velocity and acceleration profiles for the vehicle that we pull out in front. Blue color represents velocity and red acceleration.

a step size of $0.2 \mathrm{~s}$ and a spatial discretisation of $2 \mathrm{~m}$ between 0 and $100 \mathrm{~m}$ generating a total of 2500 candidate trajectory profiles. The constraints for the ego vehicle is set to correspond to a loaded truck with a maximum acceleration $a_{\max }=3 \mathrm{~m} / \mathrm{s}^{2}$, a maximum deceleration of $a_{\min }=-5 \mathrm{~m} / \mathrm{s}^{2}$ and a maximum allowed lateral acceleration of $a_{\text {lat, } \max }=$ $3.928 \mathrm{~m} / \mathrm{s}^{2}$. The sensor range is set to $180 \mathrm{~m}$ and the desired time gap, $t_{r e f}$ is set to $3 \mathrm{~s}$, the minimum time gaps are set to $t_{\text {lead,min }}=0.5 \mathrm{~s}$ and $t_{\text {follower,min }}=1.0 \mathrm{~s}$ respectively. The cost terms was set equally for the two scenarios with $w_{\text {progress }}=$ $5.0, w_{a_{\text {lat }}}=1.0, w_{\text {acc }}=0.0, w_{\text {gap }}=0.3$ and $w_{\text {interaction }}=0.5$. Finally, the parameters for the IDM used for predictions during the two scenarios are shown in Table II. The spawning parameter interval and the constraint $a_{\text {followermin }}$ are set differently for different experiments and are presented later for each experiment. The average execution time for every planning cycle during both experiments were $53.88 \mathrm{~ms}$ which indicates the possibility of running the framework at $10 \mathrm{~Hz}$ during real-time operation.

TABLE II: Prediction IDM parameters for highway merge scenario (HM) and T-junction scenario (TJ)

\begin{tabular}{c|ccccc}
\hline $\mathrm{HM}$ & $v_{0}[\mathrm{~m} / \mathrm{s}]$ & $a\left[\mathrm{~m} / \mathrm{s}^{2}\right]$ & $d_{0}[\mathrm{~m}]$ & $T[\mathrm{~s}]$ & $b\left[\mathrm{~m} / \mathrm{s}^{2}\right]$ \\
\hline & 25.0 & 3.0 & 1.0 & 2.0 & 3.0 \\
\hline $\mathrm{TJ}$ & $v_{0}[\mathrm{~m} / \mathrm{s}]$ & $a\left[\mathrm{~m} / \mathrm{s}^{2}\right]$ & $d_{0}[\mathrm{~m}]$ & $T[\mathrm{~s}]$ & $b\left[\mathrm{~m} / \mathrm{s}^{2}\right]$ \\
\hline & 13.88 & 3.0 & 1.0 & 2.0 & 3.0
\end{tabular}

\section{RESUlts}

To evaluate the performance of the presented framework the results form a simulation of the T-junction scenario is first presented before a more thorough statistical comparison with random traffic scenarios is done between the presented approach and a baseline approach where constant velocity models are assumed for other traffic participants.

The behavior of the planning framework is first evaluated in a simulation of the T-junction scenario with slow moving dense traffic. The maximum deceleration constraint $a_{\text {follower,min }}$ is set to $-3 \mathrm{~m} / \mathrm{s}^{2}$ and the ego vehicle approaches the intersection with an initial velocity of $10 \mathrm{~m} / \mathrm{s}$. The velocity and acceleration profiles for the ego vehicle and the resulting follower vehicle is recorded and presented in Fig. 4 together with snapshots of the scene at different time instances. Before the intersection the vehicle starts to slow down and smoothly decreases the velocity to nearly zero right at the stop line after approximately $17 \mathrm{~s}$. At time $t=19.1 \mathrm{~s}$ the algorithm detects a gap that is big enough to meet all safety criteria and decides to merge in front of the vehicle that gets marked with a red line. The interaction begins when the ego vehicle passes the stop line at $t=25.5 \mathrm{~s}$ and enters the oncoming lane which forces the oncoming vehicle to initiate a safe breaking with a maximum breaking effort of $-1.84 \mathrm{~m} / \mathrm{s}^{2}$ in order to leave the gap needed to complete the merge. Once the merge is complete the ego vehicle maintains a safe distance to the vehicle in front and blends in nicely in the traffic flow. Even though the time 
TABLE III: Average time to complete merge maneuver, $\bar{t}_{c}$, for traffic density $d_{i v}=90.0 \mathrm{~m}$ in TJ scenario using interaction aware motion planner.

\begin{tabular}{l|lllllll}
$a_{f, m}\left[\mathrm{~m} / \mathrm{s}^{2}\right]$ & 1.0 & 1.5 & 2.0 & 2.5 & 3.0 & 3.5 & 4.0 \\
\hline $\bar{t}_{c}[s]$ & 63.60 & 27.66 & 23.34 & 18.34 & 17.36 & 16.73 & 15.93
\end{tabular}

gap was not big enough to begin with a safe merge could still be performed by predicting the deceleration of the other vehicle needed to open the gap. In this scenario the predicted deceleration was found acceptable and a merge was initiated. A constant velocity model could not do this decision as time gaps would remain the same in the predictions and even though the other vehicle had to brake and slow down using the more advanced prediction model, the braking force were in the same range as the braking used by the ego vehicle to slow down before the stop line and should be considered safe. A video of the above scenario and several others can be found at https://youtu.be/uXKwHONE6Tk.

\section{A. Statistical Evaluation}

To get a more thorough evaluation of the performance of the two planning algorithms, the interaction aware planner and the baseline minimum time-gap planner, the merge success rate is recorded with different parameters settings for the two methods in randomly generated traffic in the HM and TJ scenarios. The algorithm is given a maximum of two minutes to perform a merge and is said to be unsuccessful if a merge has not been completed within this time limit. In the T-junction scenario an initial unsuccessful merge results in a slight deceleration to the stop line where the vehicle can safely wait for a gap that is suitable for a merge, while in the highway scenario a failed initial merge attempt can involve hard braking and a complete stop on a highway entrance ramp. If stuck on the on-ramp the sensor range of 180 meters is not enough to safely attempt a merge from zero velocity at that point due to the high velocity of oncoming traffic and will always result in an unsuccessful attempt.

Both scenarios were simulated 100 times for every parameter settings in randomly generated traffic with different density and individually randomized values from the normal distribution, $N\left(v_{r e f}, 3.5\right)$, for the desired cruising speed parameter $v_{0}$. The aggressiveness parameters for the two methods where varied with $a_{\text {follower,min }}=0.5,1.0, \ldots, 4.0 \mathrm{~m} / \mathrm{s}^{2}$ for the interaction aware planner and $t_{\text {follower,min }}=1.0,2.0, \ldots, 5.0 \mathrm{~s}$ for the baseline constant velocity planner and the results are presented in Fig. 5.

From the figure it can be seen that, in general, denser traffic requires a more aggressive setting of the planner parameters to find a successful merge strategy.

In the T-junction scenario with the lowest traffic density the baseline method has a success rate of only $22 \%$ when using the most aggressive setting of $t_{\text {follower,min }}=1.0 \mathrm{~s}$ and it drops further to only $3 \%$ with the time gap setting at $t_{\text {follower,min }}=1.5 \mathrm{~s}$. Following the more recommended 3 second rule and setting $t_{\text {follower,min }}=3.0 \mathrm{~s}$ results in
TABLE IV: Occurrence frequency of high brake situations for upstream vehicle during merge in HM scenario for interaction aware planner (IA) and baseline (BL) using $a_{\text {follower,min }}=-4 \mathrm{~m} / \mathrm{s}^{2}$ and $t_{\text {follower,min }}=1 \mathrm{~s}$, respectively.

\begin{tabular}{ccc}
$d_{i v}$ & Freq. IA. [\%] & Freq. BL. [\%] \\
\hline 50 & 1.00 & 1.00 \\
60 & 0.00 & 6.00 \\
80 & 0.00 & 3.00
\end{tabular}

unsuccessful merges for all simulated traffic densities. Even though the time gaps between the cars might be large enough for an instant merge the slow acceleration of the ego vehicle and the time it takes to finish the right turn lets the time gap shrink dramatically to the next down-stream vehicle during the turn which renders an infeasible trajectory. On the other hand, the interaction aware trajectory planner can predict that the right turn maneuver will only lead to a slight deceleration of the up-stream vehicle and can proceed. The baseline strategy can be compared to a very defensive driver that will wait until the road is completely clear before attempting the merge, while the interaction aware motion planner can be tuned to behave like a more experienced driver.

In Table III the average time the ego vehicle has to wait before a suitable gap is found in the TJ scenario is presented. The scenario is run with $d_{i v}=90 \mathrm{~m}$ and for different settings for $a_{\text {follower,min }}$ and as expected the wait time decreases with increased values for the aggressiveness parameter $a_{\text {follower,min }}$ and highlights the ability of the proposed method to balance the utility of quickly reaching the destination and the affect on other traffic.

Looking at the highway merge scenario the baseline planner only reaches acceptable success rates using the most aggressive parameter setting of $t_{\text {followermin }}=1.0 \mathrm{~s}$ while there are several settings for the proposed method that give acceptable results in all but the most dense traffic. If we compare the actual deceleration of other traffic participants caused by our own maneuver it can be seen that the mean deceleration caused by the interaction aware planner with its most aggressive setting of $a_{\text {follower,min }}=-4.0 \mathrm{~m} / \mathrm{s}^{2}$ for all simulations with the most dense traffic, $d_{i v}=50 \mathrm{~m}$, is $-1.92 \mathrm{~m} / \mathrm{s}^{2}$ while for the baseline method it is $-2.73 \mathrm{~m} / \mathrm{s}^{2}$. This indicates that the proposed method is better at choosing which of the available gaps within the sensor range to merge into and where to place the vehicle to cause the least risk for other vehicles. In Table IV the number of times the planners caused a deceleration of more than $-4 \mathrm{~m} / \mathrm{s}^{2}$, which can be considered a dangerous situation, is presented and gives an indication that the baseline algorithm is prone to more dangerous maneuvers. However with only 100 simulations for each scenario we should be careful of interpreting these results as definite and more simulations should be performed to lower the variance of these estimates.

\section{CONCLUSIONS AND FUTURE WORK}

This paper presents a trajectory planning algorithm capable of negotiating merge scenarios with dense traffic in a T-junction scenario at moderate speeds and a highway 

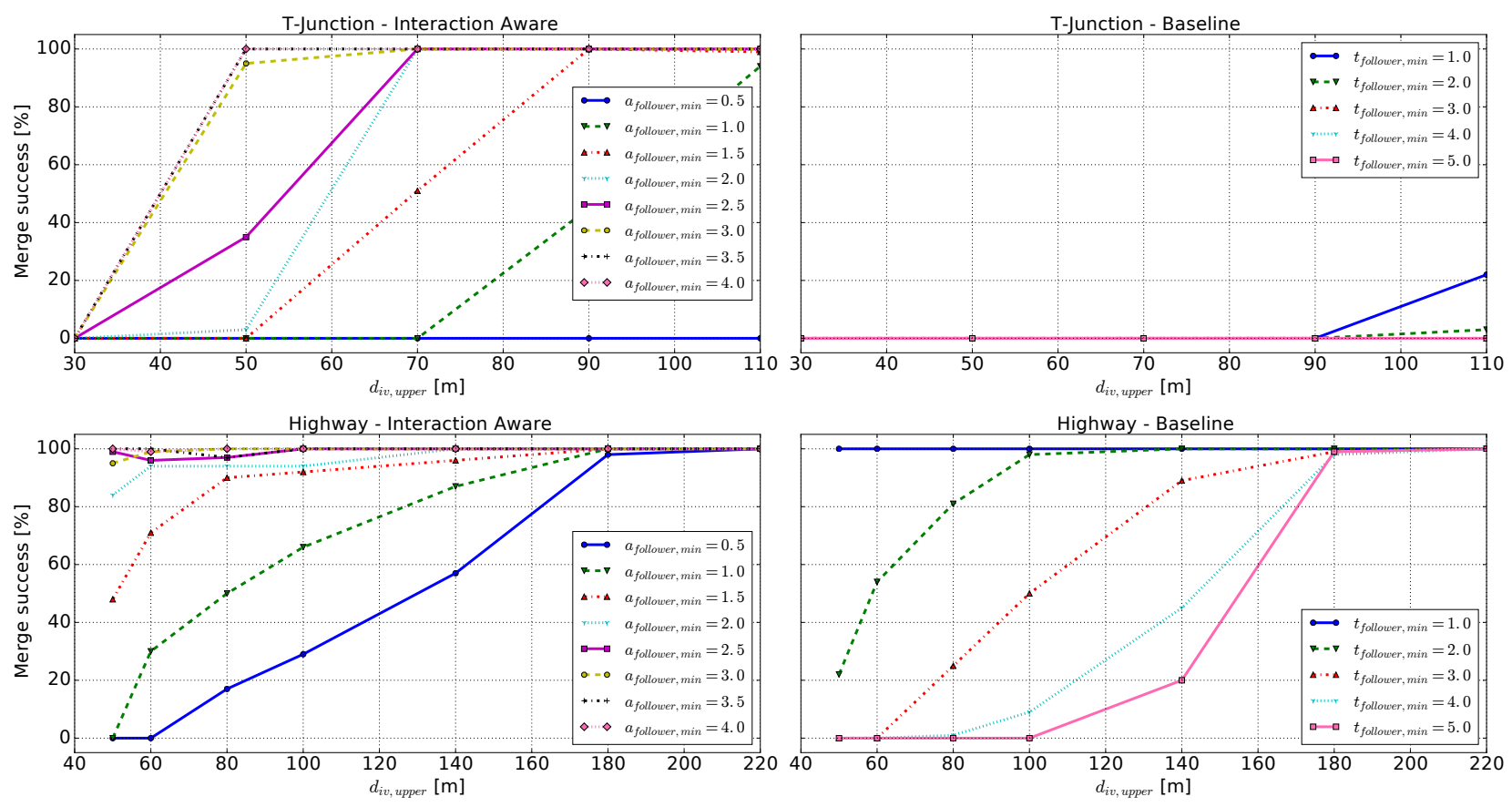

Fig. 5: Fraction of successful merge maneuvers for the HM and TJ scenarios for different $a_{\text {follower,min }}$ parameter values for the interaction aware motion planner and different minimum time gap values, $t_{\text {follower,min }}$, for the baseline planner.

merge scenario at high speeds by using a prediction engine where the affect on other traffic participants depending on our own trajectory can be predicted using the Intelligent Driver Model. The method is validated and compared to a base line method in a number of simulation experiments with randomized traffic situations in the two scenarios. By simulating the future motion for other detected vehicles in the scene, including the influence of our own vehicle, we can balance between the goals of making progress towards our destination and the risk of a manouver. The predicted decelerations from the simulations allows us to compare trajectories based on the induced deceleration on up-stream vehicles during the merge manouver and then in every planning cycle the best trajectory out of a large number of minimum jerk candidate trajectories are evaluated and chosen depending on a cost function incorporating progress, comfort and risk. In the evaluation experiments with the baseline method where other vehicles are assumed to follow a constant velocity model the proposed framework had a significantly higher success rate while still inducing a lower deceleration for the up-stream vehicles which indicates that the proposed method generally takes a better decision about which gap to merge into. Making predictions of human drivers in autonomous systems could lead to dangerous situations if the prediction is off. However, it is necessary to do in order to get any reliable operation in complex urban traffic if no infrastructure, such as V2V or V2I, exists. Future work include implementation and real world testing on one of our test platforms but also improving the predictions by individually estimating driver parameters for the IDM from sensor data and looking at methods to account for uncertainty in the tracks of other vehicles. Other interesting directions would be to include predictions of future manoeuvres of other participants e.g. lane changes or right and left turns.

\section{REFERENCES}

[1] M. Werling, T. Gindele, D. Jagszent, and L. Gröll, "A robust algorithm for handling moving traffic in urban scenarios," in Intelligent Vehicles Symposium, 2008 IEEE. IEEE, 2008, pp. 1108-1112.

[2] J. Eggert, F. Damerow, and S. Klingelschmitt, "The foresighted driver model," in Intelligent Vehicles Symposium (IV), 2015 IEEE. IEEE, 2015, pp. 322-329.

[3] M. Treiber, A. Hennecke, and D. Helbing, "Congested traffic states in empirical observations and microscopic simulations," Physical Review $E$, vol. 62 , no. 2 , p. $1805,2000$.

[4] A. Kesting, M. Treiber, and D. Helbing, "General lane-changing model mobil for car-following models," Transportation Research Record: Journal of the Transportation Research Board, 2007.

[5] D. Ferguson, T. M. Howard, and M. Likhachev, "Motion planning in urban environments," Journal of Field Robotics, vol. 25, no. 11-12, pp. $939-960,2008$.

[6] M. Montemerlo, J. Becker, S. Bhat, H. Dahlkamp, D. Dolgov, S. Ettinger, D. Haehnel, T. Hilden, G. Hoffmann, B. Huhnke, et al., "Junior: The Stanford entry in the urban challenge," Journal of field Robotics, vol. 25, no. 9, pp. 569-597, 2008.

[7] M. Werling, S. Kammel, J. Ziegler, and L. Gröll, "Optimal trajectories for time-critical street scenarios using discretized terminal manifolds," The International Journal of Robotics Research, vol. 31, no. 3, pp. 346-359, 2011.

[8] J. Ziegler, P. Bender, T. Dang, and C. Stiller, "Trajectory planning for bertha - a local, continuous method," in Intelligent Vehicles Symposium Proceedings, 2014 IEEE. IEEE, 2014, pp. 450-457.

[9] J. Wei, J. M. Dolan, and B. Litkouhi, "Autonomous vehicle social behavior for highway entrance ramp management," in Intelligent Vehicles Symposium (IV), 2013 IEEE. IEEE, 2013, pp. 201-207.

[10] A. G. Cunningham, E. Galceran, R. M. Eustice, and E. Olson, "MPDM: Multipolicy decision-making in dynamic, uncertain environments for autonomous driving," in Robotics and Automation (ICRA), 2015 IEEE International Conference on. IEEE, 2015, pp. 1670-1677.

[11] M. Liebner, F. Klanner, M. Baumann, C. Ruhhammer, and C. Stiller, "Velocity-based driver intent inference at urban intersections in the presence of preceding vehicles," Intelligent Transportation Systems Magazine, IEEE, vol. 5, no. 2, pp. 10-21, 2013. 